\title{
Comparative analysis of non-destructive methods to control fissile materials in large-size containers
}

\author{
V.F. Batyaev and S.V. Sklyarov \\ Dukhov Research Institute of Automatics (VNIIA), 127055 Moscow, Russia
}

\begin{abstract}
The analysis of various non-destructive methods to control fissile materials (FM) in large-size containers filled with radioactive waste (RAW) has been carried out. The difficulty of applying passive gamma-neutron monitoring FM in large containers filled with concreted RAW is shown. Selection of an active non-destructive assay technique depends on the container contents; and in case of a concrete or iron matrix with very low activity and low activity RAW the neutron radiation method appears to be more preferable as compared with the photonuclear one.
\end{abstract}

\section{Introduction}

According to the current regulations in the Russian Federation any radioactive wastes (RAW) must be certified before their disposal [1]. The certification of RAW containers should include determination of fissile material (FM) content inside and is aimed to ensure safety of a repository for a long time. By the present time over $500,000,000 \mathrm{~m}^{3}$ of RAW have been stockpiled in the Russian Federation. A portion of low level wastes (LLW) of different classes is $99.97 \%$ of the total RAW volume [2]. Vast majority of RAW ( $>95 \%$ ) also contains fission products which complicates FM certification.

Our recent experiments with tiny amounts of ${ }^{235} \mathrm{U}$ $(\sim 1 \mathrm{mg})$ show the possibility of its detection inside a small $60 \mathrm{~L}$ container when using a pulsed neutron generator of the ING-07T type [3]. However, the most part of RAW from Rosatom enterprises is loaded into much larger nonreturnable protective containers of NZK type [4] in the form of a hollow concrete rectangular parallelepiped of $165 \mathrm{~cm} \times 165 \mathrm{~cm} \times 134 \mathrm{~cm}$ external dimensions, having $11 \mathrm{~cm}$ thick walls, and of $3.5 \mathrm{t}$ weight, which is filled with $3.2 \mathrm{t}$ concrete matrix containing RAW. ${ }^{235} \mathrm{U}$ and other fissile nuclides content in the matrix of such container can be at the level of a few grams and less.

Since the packed RAW containers are not allowed to be disclosed later on, their subsequent certification should be carried out using just non-destructive assay methods.

The objective of the paper is to compare various nondestructive interrogation techniques potentially applicable to control tiny amounts ( $1 \mathrm{~g}$ and less) of FM in the RAW in large-sized NZK-type containers.

\section{Passive methods of FM Non-destructive control}

Both passive and active techniques can be applied as potential non-destructive methods of FM content assay. Passive methods detect an irradiation emmitted by the inspected objects without any supplementary radiation sources that are required in active techniques to provoke sought materials to emit a radiation of easily recorded and distinguished type. In case of passive methods, radionuclide composition of an inspected object is very important, because the presence of $\beta$-emitting nuclides affects negatively on metrological parameters of the control procedure.

Figure 1 shows that grams of ${ }^{235} \mathrm{U}$ cannot practically provide any significant contribution to the detector response. This is due to huge size and wall thickness of NZK container comparing to the mean free path of $186 \mathrm{keV}$ gammas. The use of the Neyman-Pearson lemma proves that such detector can discover dozens and more grams of ${ }^{235} \mathrm{U}$ depending on measurement duration.

Neutron yield from ${ }^{235} \mathrm{U}$ and ${ }^{238} \mathrm{U}$ spontaneous decay is very low $\left(\sim 10^{-2} \mathrm{n} /(\mathrm{s} \cdot \mathrm{g})\right)$, hence it is impossible to apply passive assay equipment for control of such low $\mathrm{U}$ amounts based on self neutron radiation. So it is necessary to study the potential of active methods to control FM in large sized containers.

\section{Comparison of active methods for FM control in large-size containers}

There are various active methods of FM remote detection. They can be classified according to the type of ionizing radiation (neutron, gamma, and others), technique of fission event recording (total count or coincidences), as well as a time mode of probe radiation, i.e., continuous, pulsed or cyclic. The latter mode is an irradiation with periodical significant time gaps (seconds) to measure induced activation nuclides or/and delayed neutrons. As to the coincidence technique of event recording, the best known and cheap is AWCC (Active Well Counter Coincidence) method [5], where a fission event is recorded as the coincident triggering of two or more neutron counters located around the inspected object probed by an isotopic neutron source. The disadvantage of this method is a high threshold of minimal detectable mass of the FM ( $1 \mathrm{~g}$ of ${ }^{235} \mathrm{U}$ [6]) even in case of rather small inspected object. Apparently, this method is hardly applicable for detection 


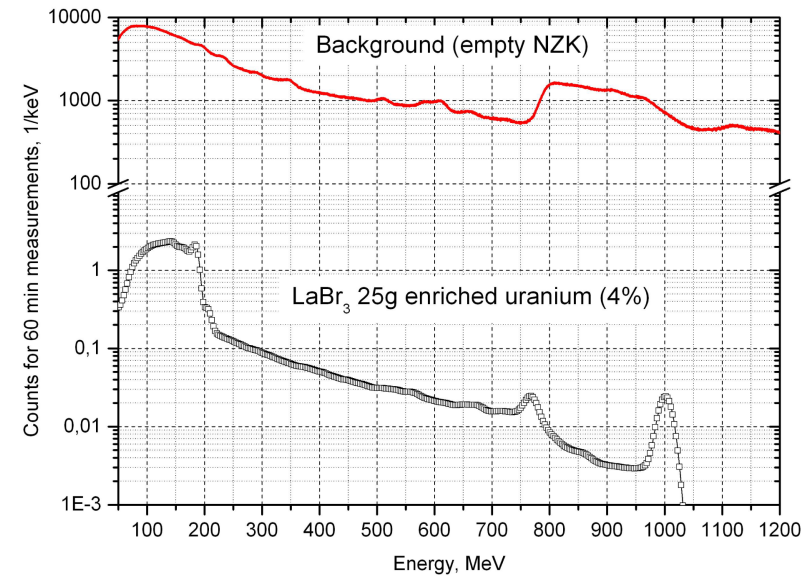

Figure 1. Results of computation simulation of $\mathrm{LaBr}_{3}(\mathrm{Ce})$ response to $25 \mathrm{~g}$ of $4 \%$ enriched uranium inside NZK container. Measurement time is $1 \mathrm{~h}, \mathrm{LaBr}_{3}(\mathrm{Ce})$ dimensions are $\varnothing 76 \times$ $76 \mathrm{~mm}$. Background spectra for the specified measurement time is also shown.

of tiny amounts $(<1 \mathrm{~g})$ of FM in a large-size container, therefore we shall limit our further review to the methods where an integral neutron radiation is recorded while the container is exposed by neutron or gamma sources.

The essential principle of the neutron-based methods is the FM response in the form of emission of prompt and delayed fission neutrons. It can be shown that the most adequate results for our purpose can be obtained if thermal neutrons are used to cause fission. Since primary and fission neutrons should be separated in time to avoid their simultaneous detection an irradiation mode must be pulsed with the time gap between pulses comparable with the lifetime of thermal neutrons, which is hundreds of $\mu \mathrm{s}$ in case of containers of NZK sizes.

The essential principle of the photonuclear method is lower value of the photofission reaction energy threshold (about $3 \mathrm{MeV}$ ) compared with the threshold of $(\gamma, n)$-reactions of the majority of structural elements ( $>8 \mathrm{MeV}$, Fig. 2). Proper choice of gamma energy is expected to cause uranium photofission reactions that would be distinguishable over a background of photoneutron reactions on structure materials of the inspected container and its matrix. In the continuous mode of a source operation a recording system shall ensure separation between detections of initial gamma photons and secondary neutrons. In cyclic mode it is possible to detect FM based on delayed neutrons. The case of using pulsed mode similar to neutron-neutron method should also be considered, when response radiation is detected in the time gaps between the source pulses.

\subsection{Computational model}

Monte Carlo simulation of an inspected object response to a probe radiation can be done with the use of mathematical model properly describing the object. In our case the NZK container was simulated by the GEANT4 MonteCarlo simulation toolkit [7]. The container was modeled with the dimensions as specified in the Introduction with ferroconcrete walls of $2.3 \mathrm{~g} / \mathrm{cm}^{3}$ density and internal content in the form of a uniform mixture of ferroconcrete matrix of $1.5 \mathrm{~g} / \mathrm{cm}^{3}$ density and the FM as $25 \mathrm{~g}$ of $4 \%$

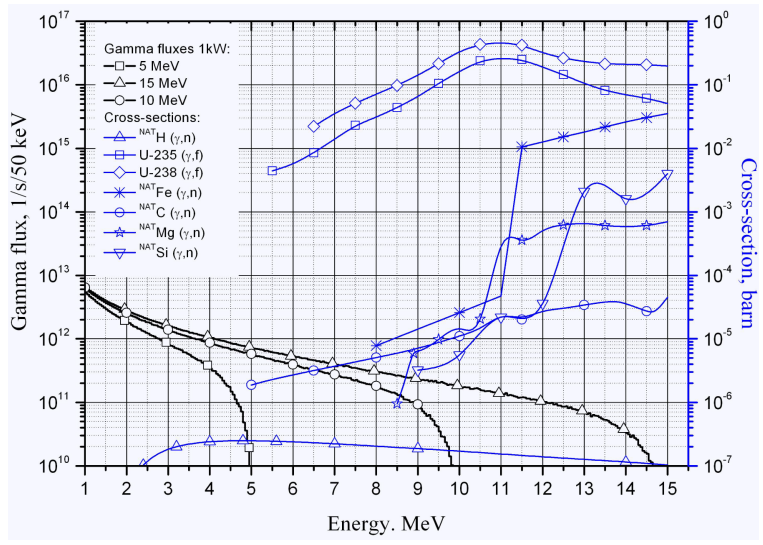

Figure 2. Bremsstrahlung spectra produced by electron beams of different energies (left axis) and photonuclear reaction crosssections (right axis).

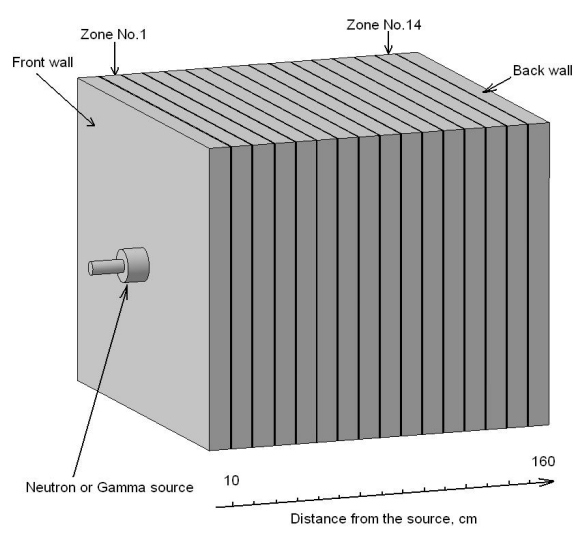

Figure 3. Schematic view of the modeled container.

enriched uranium, i.e., $1 \mathrm{~g}$ of ${ }^{235} \mathrm{U}$. Natural content of uranium in concrete was also taken into account.

It should be noted that simulation result depends on elemental composition of concrete, especially on hydrogen and carbon amount. Carbon content can vary in different types of concrete within wide range from $0 \%$ to $17 \mathrm{wt} \%$. [8], hydrogen content - from 0.1 to $1 \mathrm{wt} \%$. In case of photonuclear method, the content of these elements is important since their neutron-excess isotopes, such as ${ }^{2} \mathrm{H}$ and ${ }^{13} \mathrm{C}$, have low thresholds of $(\gamma, \mathrm{n})$-reactions (Fig. 2$)$, which can be competitive to photofission reactions. In case of neutron-neutron method, these elements can influence the values and spatial distribution of thermal neutron fluxes.

The container is divided conventionally into 16 parallel flat zones of the same thickness (Fig. 3). Radiation (n or $\gamma$ ) source is located in the middle of one of the container sides. The response radiation is supposed to be detected by neutron counters located around the container.

In case of neutron probe radiation the source was specified as an isotropic distribution of $14 \mathrm{MeV}$ neutrons (from D-T reaction) or $2.5 \mathrm{MeV}$ neutrons (from D-D reaction) with $100 \mu$ s pulse width, $400 \mathrm{~Hz}$ pulse repetition rate, and $1 \cdot 10^{9} \mathrm{n} / \mathrm{s}$ flux corresponding to the operational parameters of the ING-07 neutron generator produced at the VNIIA [9]. The simulations resulted in the rates of secondary neutrons generation due to $(n, f)-$ and $(n, 2 n)$ reactions distributed over the container zones and over the time after pulse. 


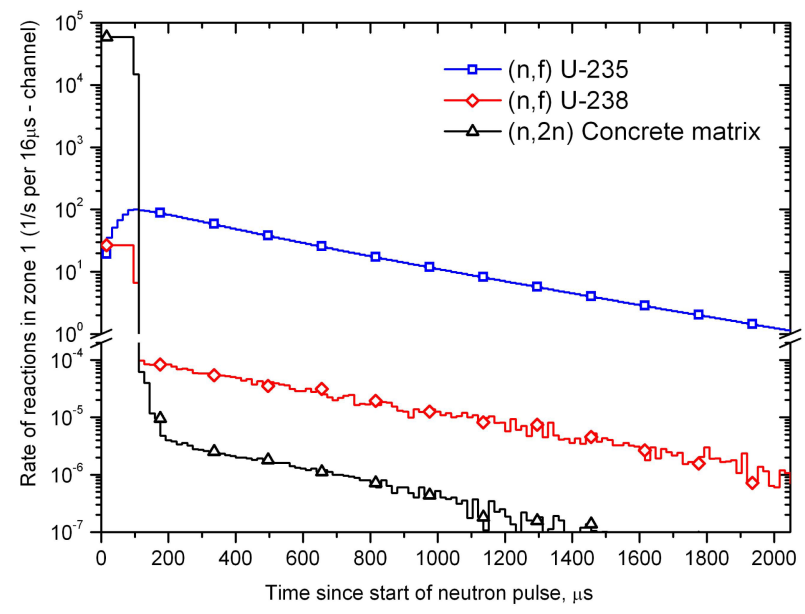

Figure 4. Time distribution of $(\mathrm{n}, \mathrm{f})$ and $(\mathrm{n}, \mathrm{xn})$ reaction rates on uranium and structural materials nuclei in zone No. 1 of the NZK container induced by $14 \mathrm{MeV}$ neutrons with $1 \cdot 10^{9} \mathrm{n} / \mathrm{s}$ flux. All distributions between pulses are exponents with decrease constant $\tau=420 \mu$ s.

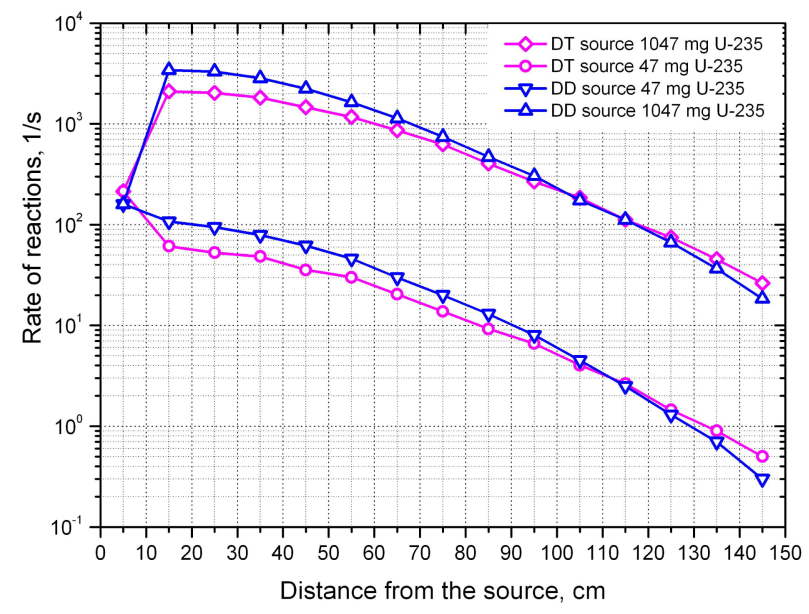

Figure 5. Depth distributions of (n,f)-reaction rates between pulses in case of DD- and DT-neutron sources of $1 \cdot 10^{9} \mathrm{n} / \mathrm{s}$ flux.

Gamma source was taken to be directed to the container center with its spectrum corresponding to bremsstrahlung of $10 \mathrm{MeV}$ initial energy electrons in tungsten (Fig. 3). Initial stage of the simulation resulted in the rates of $(\gamma, \mathrm{f})$ - and $(\gamma, \mathrm{n})$-reactions over container zones. Then the transport of the produced neutrons was simulated to obtain the rates of the above reactions during the time gap between source pulses. The results of simulations are normalized to electron beam of $1 \mathrm{~kW}$ power.

\subsection{Results of the neutron method simulation}

Figures 4 to 7 show simulation results for the rates of reactions in case of the neutron source, namely:

1. time distribution of $(\mathrm{n}, \mathrm{f})$-reactions on ${ }^{235} \mathrm{U}$ and ${ }^{238} \mathrm{U}$, as well as $(\mathrm{n}, \mathrm{xn})$-reactions on the rest elements during the pulse and gap between pulses (Fig. 4);

2. comparison of $(n, f)$-reactions distribution over the container depth for $14-$ and $2.5 \mathrm{MeV}$ sources (Fig. 5);

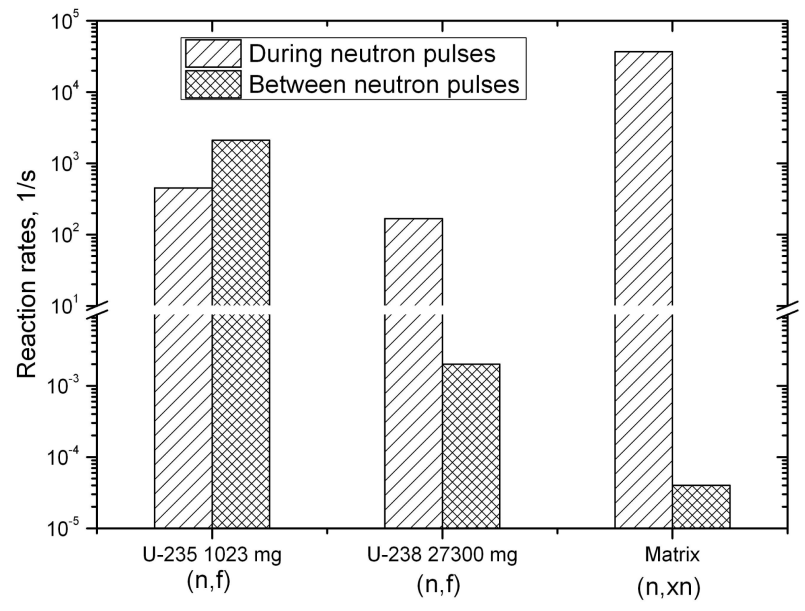

Figure 6. $(n, f)-$ and $(n, x n)$ reaction rates in zone No. 1 of the container in case of DT-neutron source of $1 \cdot 10^{9} \mathrm{n} / \mathrm{s}$ flux during the pulses and between them.

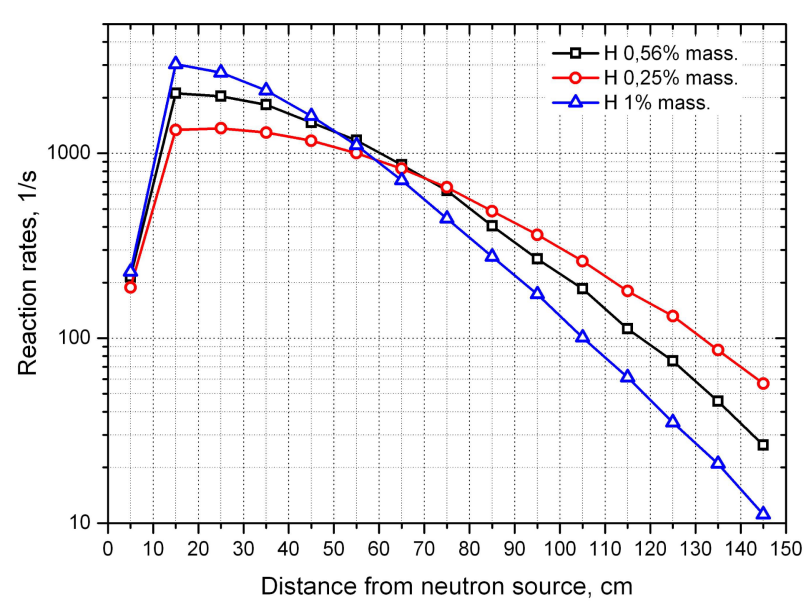

Figure 7. Depth-distributions of (n,f)-reaction rates between pulses in case of different hydrogen content in the container matrix.

3. detailing of reaction rates during the pulse and gap between pulses (Fig. 6);

4. sensitivity of $(n, f)$-reactions distribution through the container depth to hydrogen content in the container matrix (Fig. 7).

The presented results allow drawing the following conclusions:

1. Due to high values of the rates of (n,xn)-reactions on the container and matrix materials (Fig. 4), fission materials can be detected only during the gaps between pulses via prompt (i.e., in pulsed mode) and delayed neutrons (in cyclic mode too).

2. Due to the "plateau" in the first 30 to $50 \mathrm{~cm}$ of the depth distributions of (n,f)-reactions (Fig. 5), 90\% of secondary neutrons are generated in about the first meter of the container, i.e., $2 / 3$ of its content contribute to the response. In the last one third of the container the decrease constant of secondary neutrons rate is equal to $22 \mathrm{~cm}$ in case of $14 \mathrm{MeV}$ source and to $17 \mathrm{~cm}$ in case $2.5 \mathrm{MeV}$ source.

3. Due to low values of reaction rates on ${ }^{238} \mathrm{U}$ - and structural materials comparing with those on ${ }^{235} \mathrm{U}$ 


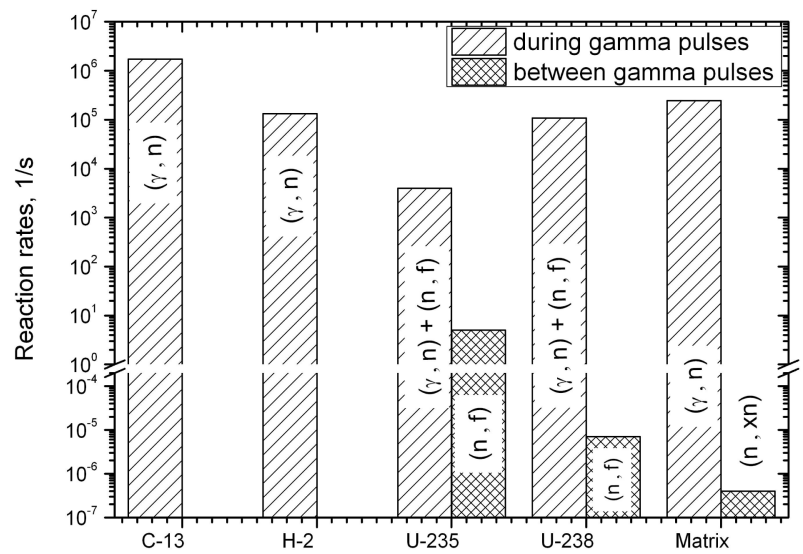

Figure 8. Rate of $(\gamma, \mathrm{n})$ - and $(\mathrm{n}, \mathrm{f})$-reactions in $1^{\text {st }}$ zone of the container induced by gamma source driven by $10 \mathrm{MeV}$ electrons of $1 \mathrm{~kW}$ power. Carbon concentration in concrete matrix was chosen to $6 \%$ mass.

(Fig. 6), reliable detection of ${ }^{235} \mathrm{U}$ is possible even if its content is comparable with natural values (i.e. tens of mg per a container).

4. Hydrogen content in the container plays a significant role both in integral amount of secondary neutrons, and in their depth distribution (Fig. 7).

\subsection{Photonuclear method simulation results}

The results of simulating the response of container elements to the exposure to gamma source are shown in Fig. 8 via the rates of primary $(\gamma, \mathrm{n})$-reactions during the pulse as well as neutron production reactions rates during time gap between pulses.

The rates of the primary $(\gamma, \mathrm{n})$-reactions (Fig. 8) show overwhelming predominance of ${ }^{13} \mathrm{C}$ in the container walls and its matrix composition as a source of the secondary neutron radiation. Taking into account above noted variability of the carbon content in the container concrete, one could estimate minimal amount of uranium that could compete with $(\gamma, \mathrm{n})$-reactions to be about hundred times higher than the value specified in this calculation, i.e., about at least $1 \mathrm{~kg}$. It should be noted that in this case the control of ${ }^{238} \mathrm{U}$ and not of ${ }^{235} \mathrm{U}$ is assumed either in continuous mode, when detecting system is capable of unmistakable distinguishing neutron and gamma detections, or in cyclic radiation mode, when delayed neutrons are detected during several seconds gaps between cycles. Note also that recording of delayed neutrons in case of large-sized containers is complicated due to a their low energy, and hence their restricted free path. Since ${ }^{238} U$ control is not quite the aim of the containers certification, then it is necessary to consider also, as it was mentioned in item 3 , the pulsed source mode, when response radiation is detected during the time gaps between pulses. Production rates of this $\left(3^{\text {rd }}\right)$ generation neutrons are shown in Fig. 8 that demonstrates considerably less intensity of these neutrons as compared to those ones in produced the neutron source case. Comparison of ${ }^{235} \mathrm{U}(\mathrm{n}, \mathrm{f})$-rates presented in Fig. 6 and Fig. 8 shows that photonuclear technique provides at least two orders of magnitude less amount of such neutrons compared with neutron based method. Though the neutron source has an evident advantage as compared to gamma, the latter shall not be neglected entirely, since it has a number of practical advantages comparing with neutrons, such as longer lifetime, no induced activity, ease of operation, etc.

\section{Conclusion}

Different non-destructive methods of FM content control in large-sized containers with concreted RAW have been compared. An application of passive methods to inspect large containers is shown as impossible due to tiny amount of FM ( $1 \mathrm{~g}$ and less) to be detected and high absorption capacity both of the container itself and the filling matrix.

FM response to the exposure of neutron (2.5 and $14 \mathrm{MeV})$ and gamma sources $(10 \mathrm{MeV})$ on the container were estimated. The neutron methods are shown to be more preferable for control of large-sized containers as compared to photonuclear method due to higher values of radiation response that allow controlling small amounts ( $1 \mathrm{~g}$ and less of ${ }^{235} \mathrm{U}$ ), and due to capability of determining not only ${ }^{235} \mathrm{U}$ based on prompt neutrons but ${ }^{238} \mathrm{U}$ as well based on delayed neutrons. At the same time potential advantages of the photonuclear method are also marked in the view of its practical implementation for control of containers with higher $U$ content (over $1 \mathrm{~kg}$ per a container).

\section{References}

[1] Federal Law 190-fz, www.en.special.kremlin. ru/ catalog/ keywords/83/events/11923

[2] www.norao.ru/upload/norao_brosh_na_site1. pdf (in Russian)

[3] V.F. Batyaev, O.V. Bochkarev, S.V. Sklyarov, Int. Jour. of Mod. Phys. 27, 1460130 (2014)

[4] NZK-MR concrete container, http://www.radon. ru/eng/activities/radwaste management/conditioning/

[5] Goddard B. et al., Nucl. Instr. \& Meth. in Phys. Res. A 830, 256-264 (2016)

[6] D. Reilly et al., Passive Nondestructive Assay of Nuclear Materials (U.S. Nuclear Regulatory Commission, Washington DC, 1991) NUREG/CR5550

[7] S. Agostineli, J. Allison et al. Nucl. Instr. \& Meth. in Phys. Res., A 506, 250-303 (2003)

[8] A.M. Neville, Properties of concrete, 2011

[9] Dukhov Research Institute of Automatics (VNIIA), http://www . vniia.ru/eng/ng/docs/prospect_ element_eng.pdf 